\title{
On Best Proximity Points under the $P$-Property on Partially Ordered Metric Spaces
}

\author{
Mohamed Jleli, ${ }^{1}$ Erdal Karapinar, ${ }^{2}$ and Bessem Samet ${ }^{1}$ \\ ${ }^{1}$ Department of Mathematics, College of Science, King Saud University, P.O. Box 2455, Riyadh 11451, Saudi Arabia \\ ${ }^{2}$ Department of Mathematics, Atilim University, Incek, 06836 Ankara, Turkey
}

Correspondence should be addressed to Erdal Karapinar; erdalkarapinar@yahoo.com

Received 11 May 2013; Accepted 16 June 2013

Academic Editor: Calogero Vetro

Copyright (c) 2013 Mohamed Jleli et al. This is an open access article distributed under the Creative Commons Attribution License, which permits unrestricted use, distribution, and reproduction in any medium, provided the original work is properly cited.

Very recently, Abkar and Gabeleh (2013) observed that some best proximity point results under the $P$-property can be obtained from the same results in fixed-point theory. In this paper, motivated by this mentioned work, we show that the most best proximity point results on a metric space endowed with a partial order (under the $P$-property) can be deduced from existing fixed-point theorems in the literature. We present various model examples to illustrate this point of view.

\section{Introduction}

Let $A$ and $B$ be two nonempty subsets of a metric space $(X, d)$. Through this paper, we will use the following notations:

$$
\begin{aligned}
d(A, B) & :=\inf \{d(a, b): a \in A, b \in B\}, \\
A_{0} & :=\{a \in A: d(a, b)=d(A, B) \text { for some } b \in B\}, \\
B_{0} & :=\{b \in B: d(a, b)=d(A, B) \text { for some } a \in A\} .
\end{aligned}
$$

Definition 1. An element $x^{*} \in A$ is said to be a best proximity point of the nonself-mapping $T: A \rightarrow B$ if and only if it satisfies the condition that

$$
d\left(x^{*}, T x^{*}\right)=d(A, B) .
$$

The notion of $P$-property was introduced in [1] as follows.

Definition 2. Let $(A, B)$ be a pair of nonempty subsets of a metric space $(X, d)$ with $A_{0} \neq \emptyset$. Then the pair $(A, B)$ is said to have the $P$-property if and only if

$$
\left.\begin{array}{l}
d\left(x_{1}, y_{1}\right)=d(A, B) \\
d\left(x_{2}, y_{2}\right)=d(A, B)
\end{array}\right\} \Longrightarrow d\left(x_{1}, x_{2}\right)=d\left(y_{1}, y_{2}\right)
$$

where $x_{1}, x_{2} \in A$ and $y_{1}, y_{2} \in B$.
Various best proximity point results for different classes of nonself-mappings under the $P$-property were established recently (see, e.g., [1-6] and references therein). Very recently, Abkar and Gabeleh [7] observed that some best proximity point results under the $P$-property can be obtained from the same results in fixed-point theory. The main purpose of this paper is to show that in many cases best proximity point results (under the $P$-property) on partially ordered metric spaces can also be deduced from the corresponding fixed-point theorems. We present various model examples to illustrate this point of view.

\section{Preliminaries}

As model examples, we will consider some known fixed-point theorems in the framework of partially ordered metric spaces.

At first, we need to introduce two classes of real functions. Denote by $\Psi$ the class of all functions $\psi:[0,+\infty[\rightarrow[0,+\infty[$ satisfying the following conditions:

$$
\begin{aligned}
& \left(\Psi_{1}\right) \psi \text { is continuous and nondecreasing, } \\
& \left(\Psi_{2}\right) \psi^{-1}(\{0\})=\{0\} .
\end{aligned}
$$

We denote by $\Phi$ the set of functions $\varphi:[0,+\infty[\rightarrow[0,+\infty[$ satisfying the following conditions:

$$
\begin{aligned}
& \left(\Phi_{1}\right) \varphi \text { is nondecreasing, } \\
& \left(\Phi_{2}\right) \lim _{n \rightarrow+\infty} \varphi^{n}(t)=0 \text { for each } t>0 .
\end{aligned}
$$


Through this paper, $\mathbb{N}$ denotes the set of all natural numbers, and $\mathbb{N}^{*}=\mathbb{N} \backslash\{0\}$.

The following concepts will be useful later.

Definition 3. Let $(X, \preceq)$ be a partially ordered set and $T$ : $X \rightarrow X$ be a giving mapping. We say that $T$ is nondecreasing (with respect to $\preceq$ ) if and only if

$$
x, y \in X, \quad x \preceq y \Longrightarrow T x \preceq T y .
$$

Definition 4 . Let $(X, \preceq)$ be a partially ordered set and $\left\{x_{n}\right\}$ be a sequence in $X$. We say that $\left\{x_{n}\right\}$ is nondecreasing (with respect to $\preceq$ ) if and only if $x_{n} \preceq x_{n+1}$ for all $n \in \mathbb{N}$.

Definition 5 . Let $(X, \preceq)$ be a partially ordered set and $d$ be a metric on $X$. We say that $(X, \preceq, d)$ is regular if and only if the following condition holds:

$$
\left.\begin{array}{c}
\left\{x_{n}\right\} \subset X \text { is nondecreasing } \\
\lim _{n \rightarrow+\infty} d\left(x_{n}, x\right)=0, x \in X
\end{array}\right\} \Longrightarrow x_{n} \preceq x, \quad \forall n \in \mathbb{N}
$$

Definition 6 . Let $(X, \preceq)$ be a partially ordered set. We say that $(X, \preceq)$ is directed if and only if

$$
\forall(x, y) \in X \times X, \quad \exists z \in X \mid(x \preceq z) \wedge(y \preceq z) .
$$

In [8], Harjani and Sadarangani proved the following fixed point result.

Theorem 7. Let $(X, \preceq)$ be a partially ordered set and suppose that there exists a metric $d$ on $X$ such that $(X, d)$ is a complete metric space. Let $T: X \rightarrow X$ be a giving mapping. Suppose that the following conditions hold:

(i) $T$ is continuous or $(X, \preceq, d)$ is regular,

(ii) $T$ is nondecreasing,

(iii) there exists $x_{0} \in X$ such that $x_{0} \preceq T x_{0}$,

(iv) for all $x, y \in X$ such that $x \preceq y$, we have

$$
\psi(d(T x, T y)) \leq \psi(d(x, y))-\varphi(d(x, y)),
$$

where $\psi, \phi \in \Psi$.

Then $T$ has a fixed point. Moreover, the sequence $\left\{T^{n} x_{0}\right\}$ converges to this fixed point. If, in addition, $(X, \preceq)$ is directed, we have uniqueness of the fixed point. result.

In [9], Agarwal et al. established the following fixed point

Theorem 8. Let $(X, \preceq)$ be a partially ordered set and suppose that there exists a metric $d$ in $X$ such that $(X, d)$ is a complete metric space. Let $T: X \rightarrow X$ be a giving mapping. Suppose that the following conditions hold:

(i) $T$ is continuous or $(X, \preceq, d)$ is regular,

(ii) $T$ is nondecreasing, (iii) there exists $x_{0} \in X$ such that $x_{0} \preceq T x_{0}$,

(iv) for all $x, y \in X$ such that $x \preceq y$, we have

$$
\begin{aligned}
& d(T x, T y) \\
& \leq \varphi(\max \{d(x, y), d(x, T x), d(y, T y), \\
& \left.\left.\frac{d(x, T y)+d(y, T x)}{2}\right\}\right),
\end{aligned}
$$

where $\varphi \in \Phi$.

Then $T$ has a fixed point. Moreover, the sequence $\left\{T^{n} x_{0}\right\}$ converges to this fixed point. in $[10]$.

The above result was subsequently extended by Turinici

Through this paper, $(A, B)$ is a pair of nonempty subsets of a metric space $(X, d)$, where $X$ is endowed with a partial order $\preceq$. We denote

$$
\begin{aligned}
d(A, B) & :=\inf \{d(a, b): a \in A, b \in B\}, \\
A_{0} & :=\{a \in A: d(a, b)=d(A, B) \text { for some } b \in B\}, \\
B_{0} & :=\{b \in B: d(a, b)=d(A, B) \text { for some } a \in A\} .
\end{aligned}
$$

Definition 9. An element $x^{*} \in A$ is said to be a best proximity point of the nonself-mapping $T: A \rightarrow B$ if and only if

$$
d\left(x^{*}, T x^{*}\right)=d(A, B) .
$$

The notion of $P$-property was introduced in [1] as follows.

Definition 10. Let $(A, B)$ be a pair of nonempty subsets of a metric space $(X, d)$ with $A_{0} \neq \emptyset$. Then the pair $(A, B)$ is said to have the $P$-property if and only if

$$
\left.\begin{array}{l}
d\left(x_{1}, y_{1}\right)=d(A, B) \\
d\left(x_{2}, y_{2}\right)=d(A, B)
\end{array}\right\} \Longrightarrow d\left(x_{1}, x_{2}\right)=d\left(y_{1}, y_{2}\right)
$$

where $x_{1}, x_{2} \in A$ and $y_{1}, y_{2} \in B$.

In [11], the authors introduced the concept of proximally increasing mappings.

Definition 11. A nonself-mapping $T: M \subseteq A \rightarrow B$ is said to be proximally increasing if and only if

$$
\left.\begin{array}{l}
y_{1} \preceq y_{2} \\
d\left(x_{1}, T y_{1}\right)=d(A, B) \\
d\left(x_{2}, T y_{2}\right)=d(A, B)
\end{array}\right\} \Longrightarrow x_{1} \preceq x_{2},
$$

where $x_{1}, x_{2} \in A, y_{1}, y_{2} \in M$

Definition 12. Let $(M, N)$ be a pair of nonempty subsets of $X$. A mapping $I: M \rightarrow N$ is said to be an isometry if and only if

$$
d(I x, I y)=d(x, y), \quad \forall x, y \in M
$$


The following lemmas will be useful later.

Lemma 13. Let $(M, N)$ be a pair of nonempty subsets of $X$. Let $I: M \rightarrow N$ be a bijective mapping. Then I is an isometry if and only if $I^{-1}$ is an isometry.

Proof. Suppose that $I$ is an isometry. Let $z, t \in N, x=I^{-1} z$ and $y=I^{-1} t$. Since $I$ is an isometry, we have

$$
d(I x, I y)=d(x, y)
$$

that is,

$$
d(z, t)=d\left(I^{-1} z, I^{-1} t\right)
$$

Then $I^{-1}$ is an isometry.

Lemma 14. Consider two nonself-mappings $\widetilde{T}: A_{0} \rightarrow B_{0}$ and $I: A_{0} \rightarrow B_{0}$. Suppose that the following conditions hold:

(i) $\widetilde{T}$ is proximally increasing,

(ii) $I: A_{0} \rightarrow B_{0}$ is bijective,

(iii) $d(x, I x)=d(A, B)$ for all $x \in A_{0}$.

Then the mapping $I^{-1} \widetilde{T}: A_{0} \rightarrow A_{0}$ is nondecreasing (with respect to $\preceq$ ).

Proof. Let $x, y \in A_{0}$ such that $x \preceq y$. We have

$$
\begin{gathered}
x \preceq y, \\
d\left(I^{-1} \widetilde{T} x, \widetilde{T} x\right)=d\left(I^{-1} \widetilde{T} x, I\left(I^{-1} \widetilde{T} x\right)\right) \\
=d(A, B)(\text { from (iii)), } \\
d\left(I^{-1} \widetilde{T} y, \widetilde{T} y\right)=d\left(I^{-1} \widetilde{T} y, I\left(I^{-1} \widetilde{T} y\right)\right)=d(A, B) .
\end{gathered}
$$

Since $\widetilde{T}$ is proximally increasing, we get that $I^{-1} \widetilde{T} x \preceq I^{-1} \widetilde{T} y$.

Lemma 15. Consider two nonself-mappings $\widetilde{T}: A_{0} \rightarrow B_{0}$ and $I: A_{0} \rightarrow B_{0}$. Suppose that the following conditions hold:

(i) $I: A_{0} \rightarrow B_{0}$ is bijective,

(ii) $d(x, I x)=d(A, B)$ for all $x \in A_{0}$,

(iii) there exist $x_{0}, x_{1} \in A_{0}$ such that $d\left(x_{1}, \widetilde{T} x_{0}\right)=d(A, B)$ and $x_{0} \preceq x_{1}$,

(iv) the pair $(A, B)$ satisfies the P-property.

Then $x_{0} \preceq I^{-1} \widetilde{T} x_{0}$.

Proof. Using (ii) with $x=x_{1}$, we have $d\left(x_{1}, I x_{1}\right)=d(A, B)$. From condition (iii), we have $d\left(x_{1}, \widetilde{T} x_{0}\right)=d(A, B)$. It follows from the $P$ property (condition (iv)) that $d\left(I x_{1}, \widetilde{T} x_{0}\right)=0$, which implies that $I x_{1}=\widetilde{T} x_{0}$. From (i) and (iii), we obtain that $x_{0} \preceq x_{1}=I^{-1} \widetilde{T} x_{0}$.

Lemma 16. Let $(A, B)$ be a pair of nonempty closed subsets of a complete metric space $(X, d)$ such that $A_{0}$ is nonempty and $(A, B)$ has the $P$ property. Then $\left(A_{0}, B_{0}\right)$ is a closed pair of subsets of $X$.
The proof of Lemma 16 can be found in [5].

Lemma 17. Let $(A, B)$ be a pair of nonempty closed subsets of a metric space $(X, d)$ such that $A_{0}$ is nonempty. Assume that the pair $(A, B)$ has the $P$ property. Then there exists a bijective isometry $I: A_{0} \rightarrow B_{0}$ such that

$$
d(x, I x)=d(A, B), \quad \forall x \in A_{0} .
$$

The proof of Lemma 17 can be found in [7].

\section{Discussions and Results}

In [11], the authors established the following best proximity point result.

Theorem 18. Let $X$ be a nonempty set such that $(X, \preceq)$ is a partially ordered set and $(X, d)$ is a complete metric space. Let $(A, B)$ be a pair of nonempty closed subsets of the metric space $(X, d)$ such that $A_{0} \neq \emptyset$. Let $T: A \rightarrow B$ be a nonself-mapping. Suppose that the following conditions hold:

(i) $T\left(A_{0}\right) \subseteq B_{0}$ and $(A, B)$ satisfy the P property,

(ii) $T$ is a continuous on $A$,

(iii) $T$ is proximally increasing,

(iv) there exist $x_{0}, x_{1} \in A_{0}$ such that

$$
d\left(x_{1}, T x_{0}\right)=d(A, B), \quad x_{0} \preceq x_{1},
$$

(v) for all $x, y \in A$ such that $x \preceq y$, we have

$$
\psi(d(T x, T y)) \leq \psi(d(x, y))-\varphi(d(x, y)),
$$

where $\psi, \varphi \in \Psi$.

Then there exists an element $x^{*} \in A$ such that

$$
d\left(x^{*}, T x^{*}\right)=d(A, B) .
$$

Further, the sequence $\left\{x_{n}\right\}$, defined by

$$
d\left(x_{n+1}, T x_{n}\right)=d(A, B), \quad \forall n \in \mathbb{N},
$$

converges to the element $x^{*}$.

We shall prove that the best proximity point result given by Theorem 18 is a consequence of the fixed point result given by Theorem 7 .

3.1. Proof of Theorem 18. Denote by $\widetilde{T}$ the restriction of the mapping $T$ to the subset $A_{0}$ of $A$. Since $T\left(A_{0}\right) \subseteq B_{0}$, we have $\widetilde{T}: A_{0} \rightarrow B_{0}$. From Lemma 17, there exists a bijective isometry $I: A_{0} \rightarrow B_{0}$ such that

$$
d(x, I x)=d(A, B), \quad \forall x \in A_{0} .
$$

Consider the self-mapping $S:=I^{-1} \widetilde{T}: A_{0} \rightarrow A_{0}$. We shall prove that the mapping $S$ satisfies all the conditions of Theorem 7. 
Claim 1. $\left(A_{0}, d\right)$ is complete.

From Lemma $16, A_{0}$ is a closed subset of the complete metric space $(X, d)$. Then $\left(A_{0}, d\right)$ is complete.

Claim 2. $S$ is continuous and nondecreasing mapping.

From Lemma $13, I^{-1}$ is an isometry, so $I^{-1}$ is continuous on $B_{0}$. Since $T$ is continuous on $A, \widetilde{T}$ is continuous on $A_{0}$. Thus, the self-mapping $S=I^{-1} \widetilde{T}$ is continuous on $A_{0}$. On the other hand, since $T$ is proximally increasing, then $\widetilde{T}$ is also a proximally increasing mapping. Now, our claim follows immediately from Lemma 14.

Claim 3. There exists $a \in A_{0}$ such that $a \preceq S a$.

We have

$$
\begin{aligned}
& d\left(x_{1}, T x_{0}\right)=d(A, B), \\
& d\left(x_{1}, I x_{1}\right)=d(A, B) .
\end{aligned}
$$

Using the $P$ property, we obtain that $d\left(T x_{0}, I x_{1}\right)=0$, which implies that $\widetilde{T} x_{0}=T x_{0}=I x_{1}$, that is, $x_{1}=I^{-1} \widetilde{T} x_{0}=S x_{0}$. Since $x_{0} \preceq x_{1}$, our claim holds with $a=x_{0}$.

Claim 4. The mapping $S$ satisfies condition (iv) of Theorem 7.

Let $x, y \in A_{0}$ such that $x \preceq y$. Since $I^{-1}$ is an isometry, it follows from condition $(\mathrm{v})$ that

$$
\begin{aligned}
\psi(d(S x, S y)) & =\psi\left(d\left(I^{-1} \widetilde{T} x, I^{-1} \widetilde{T} y\right)\right)=\psi(d(T x, T y)) \\
& \leq \psi(d(x, y))-\varphi(d(x, y)) .
\end{aligned}
$$

This proves our claim.

Now, the mapping $S$ satisfies all the conditions of Theorem 7. We deduce that $S$ has a fixed point $x^{*} \in A_{0}$, and the sequence $\left\{z_{n}\right\} \subset A_{0}$, defined by

$$
z_{0}=x_{0}, \quad z_{n+1}=S z_{n}, \quad \forall n \in \mathbb{N},
$$

converges to $x^{*}$

We claim that $x^{*}$ is a best proximity point of $T$. Indeed, we have

$$
0=d\left(x^{*}, S x^{*}\right)=d\left(x^{*}, I^{-1} \widetilde{T} x^{*}\right)
$$

which implies that $x^{*}=I^{-1} \widetilde{T} x^{*}$, that is, $I x^{*}=\widetilde{T} x^{*}=T x^{*}$. From $d\left(x^{*}, I x^{*}\right)=d(A, B)$, we obtain that $d\left(x^{*}, T x^{*}\right)=$ $d(A, B)$. Then $x^{*}$ is a best proximity point of $T$.

Let $\left\{x_{n}\right\} \subset A_{0}$ be the sequence defined by

$$
d\left(x_{n+1}, T x_{n}\right)=d(A, B), \quad \forall n \in \mathbb{N} .
$$

Since

$$
d\left(x_{n+1}, I x_{n+1}\right)=d(A, B), \quad \forall n \in \mathbb{N},
$$

from the $P$ property, we get that

$$
I x_{n+1}=T x_{n}, \quad \forall n \in \mathbb{N},
$$

which implies that

$$
x_{n+1}=S x_{n}, \quad \forall n \in \mathbb{N} \text {. }
$$

It follows from (25) and (30) that $\left\{x_{n}\right\}=\left\{z_{n}\right\} \rightarrow x^{*}$ as $n \rightarrow$ $+\infty$. This makes end to the proof of Theorem 18 .

Remark 19. In Theorem 18, the authors considered only the continuous case. However, from Theorem 7, we can also remove the continuity assumption of $T$ and replace it by the regularity assumption of $\left(A_{0}, \preceq, d\right)$. Moreover, by assuming that $\left(A_{0}, \preceq\right)$ is directed, we obtain uniqueness of the best proximity point.

3.2. Additional Result. Using the same techniques, we can obtain a best proximity version of Theorem 8 .

Theorem 20. Let $X$ be a nonempty set such that $(X, \preceq)$ is a partially ordered set and $(X, d)$ is a complete metric space. Let $(A, B)$ be a pair of nonempty closed subsets of the metric space $(X, d)$ such that $A_{0} \neq \emptyset$. Let $T: A \rightarrow B$ be a nonself-mapping. Suppose that the following conditions hold:

(i) $T\left(A_{0}\right) \subseteq B_{0}$ and $(A, B)$ satisfy the $P$ property,

(ii) $T$ is continuous on $A_{0}$ or $\left(A_{0}, \preceq, d\right)$ is regular,

(iii) $T$ is proximally increasing,

(iv) there exist $x_{0}, x_{1} \in A_{0}$ such that

$$
d\left(x_{1}, T x_{0}\right)=d(A, B), \quad x_{0} \preceq x_{1},
$$

(v) for all $x, y \in A$ such that $x \preceq y$, we have

$$
d(T x, T y) \leq \varphi\left(M_{T}(A, B, x, y)\right),
$$

where $\varphi \in \Phi$ and

$$
\begin{aligned}
& M_{T}(A, B, x, y) \\
& :=\max \{d(x, y), d(x, T x)-d(A, B), d(y, T y) \\
& \left.\quad-d(A, B), \frac{d(x, T y)+d(y, T x)}{2}-d(A, B)\right\} .
\end{aligned}
$$

Then there exists an element $x^{*} \in A$ such that

$$
d\left(x^{*}, T x^{*}\right)=d(A, B)
$$

Further, the sequence $\left\{x_{n}\right\}$, defined by

$$
d\left(x_{n+1}, T x_{n}\right)=d(A, B), \quad \forall n \in \mathbb{N},
$$

converges to the element $x^{*}$.

Proof. We continue to use the same notations as in the proof of Theorem 18. Following the proof of Theorem 18, we have only to check that the self-mapping $S=I^{-1} \widetilde{T}: A_{0} \rightarrow A_{0}$ 
satisfies condition (iv) of Theorem 8. Indeed, let $x, y \in A_{0}$ such that $x \leq y$, we have

$$
\begin{aligned}
d(S x, S y) & =d\left(I^{-1} \widetilde{T} x, I^{-1} \widetilde{T} y\right)=d(T x, T y) \\
& \leq \varphi\left(M_{T}(A, B, x, y)\right) .
\end{aligned}
$$

On the other hand, one has the evaluation

$$
\begin{aligned}
M_{T}(A, B, x, y) & \\
=\max & \{d(x, y), d(x, T x)-d(A, B), d(y, T y) \\
& \left.\quad d(A, B), \frac{d(x, T y)+d(y, T x)}{2}-d(A, B)\right\} \\
= & \max \{d(x, y), \alpha, \beta, \gamma\},
\end{aligned}
$$

where we denoted

$$
\begin{aligned}
& \alpha:=d(x, \widetilde{T} x)-d(A, B), \\
& \beta:=d(y, \widetilde{T} y)-d(A, B), \\
& \gamma:=\frac{d(x, \widetilde{T} y)+d(y, \widetilde{T} x)}{2}-d(A, B) .
\end{aligned}
$$

\section{Estimation of $\alpha$.}

We have

$$
\begin{aligned}
\alpha & =d(x, \widetilde{T} x)-d(A, B) \\
& \leq d(x, S x)+d(S x, \widetilde{T} x)-d(A, B) \\
& =d(x, S x)+d\left(I^{-1} \widetilde{T} x, \widetilde{T} x\right)-d(A, B) \\
& =d(x, S x)+d\left(I^{-1} \widetilde{T} x, I\left(I^{-1} \widetilde{T} x\right)\right)-d(A, B) \\
& =d(x, S x)+d(A, B)-d(A, B)(\text { from }(22)) \\
& =d(x, S x) .
\end{aligned}
$$

\section{Estimation of $\beta$.}

We have

$$
\begin{aligned}
\beta & =d(y, \widetilde{T} y)-d(A, B) \\
& \leq d(y, S y)+d(S y, \widetilde{T} y)-d(A, B) \\
& =d(y, S y)+d\left(I^{-1} \widetilde{T} y, \widetilde{T} y\right)-d(A, B) \\
& =d(y, S y)+d\left(I^{-1} \widetilde{T} y, I\left(I^{-1} \widetilde{T} y\right)\right)-d(A, B) \\
& =d(y, S y) .
\end{aligned}
$$

Estimation of $\gamma$.

We have

$$
\begin{aligned}
\gamma= & \frac{d(x, \widetilde{T} y)+d(y, \widetilde{T} x)}{2}-d(A, B) \\
\leq & \frac{d(x, S y)+d(S y, \widetilde{T} y)+d(y, S x)+d(S x, \widetilde{T} x)}{2} \\
& -d(A, B) \\
= & \frac{d(x, S y)+d\left(I^{-1} \widetilde{T} y, \widetilde{T} y\right)+d(y, S x)+d\left(I^{-1} \widetilde{T} x, \widetilde{T} x\right)}{2} \\
& -d(A, B) \\
= & \frac{d(x, S y)+d(A, B)+d(y, S x)+d(A, B)}{2} \\
& -d(A, B) \\
= & \frac{d(x, S y)+d(y, S x)}{2} .
\end{aligned}
$$

Now, we deduce that

$$
\begin{gathered}
M_{T}(A, B, x, y) \\
\leq \max \{d(x, y), d(x, S x), d(y, S y), \\
\left.\frac{d(x, S y)+d(y, S x)}{2}\right\} .
\end{gathered}
$$

Since $\varphi$ is nondecreasing, we get that

$$
\begin{array}{r}
\varphi\left(M_{T}(A, B, x, y)\right) \\
\leq \varphi(\max \{d(x, y), d(x, S x), d(y, S y), \\
\left.\left.\frac{d(x, S y)+d(y, S x)}{2}\right\}\right) .
\end{array}
$$

Finally, it follows from (36) and (43) that

$$
\begin{gathered}
d(S x, S y) \\
\leq \varphi(\max \{d(x, y), d(x, S x), d(y, S y), \\
\left.\left.\frac{d(x, S y)+d(y, S x)}{2}\right\}\right) .
\end{gathered}
$$

Thus, we proved that the mapping $S$ satisfies condition (iv) of Theorem 8. Applying Theorem 8, we obtain the existence of a fixed point of $S$. The rest of the proof is exactly like that of Theorem 18, so we omit it. 


\section{Acknowledgment}

The authors would like to extend their sincere appreciation to the Deanship of Scientific Research at King Saud University for its funding of this research through the Research Group Project no RGP-VPP-237.

\section{References}

[1] V.S. Raj, "A best proximity point theorem for weakly contractive non-self-mappings," Nonlinear Analysis. Theory, Methods \& Applications, vol. 74, no. 14, pp. 4804-4808, 2011.

[2] A. Abkar and M. Gabeleh, "The existence of best proximity points for multivalued non-self-mappings," Revista de la Real Academia de Ciencias Exactas, Fisicas y Naturales. Serie A. Matematicas, 2013.

[3] A. Abkar and M. Gabeleh, "Global optimal solutions of noncyclic mappings in metric spaces," Journal of Optimization Theory and Applications, vol. 153, no. 2, pp. 298-305, 2012.

[4] A. Abkar and M. Gabeleh, "Best proximity points of non-self mappings," TOP, pp. 1-9, 2012.

[5] M. Gabeleh, "Proximal weakly contractive and proximal nonexpansive non-self-mappings in metric and Banach spaces," Journal of Optimization Theory and Applications, 2013.

[6] B. Samet, "Some results on best proximity points," Journal of Optimization Theory and Applications, 2013.

[7] A. Abkar and M. Gabeleh, "A note on some best proximity point theorems proved under P-property," http://arxiv. org/abs/1301.7147.

[8] J. Harjani and K. Sadarangani, "Generalized contractions in partially ordered metric spaces and applications to ordinary differential equations," Nonlinear Analysis. Theory, Methods \& Applications, vol. 72, no. 3-4, pp. 1188-1197, 2010.

[9] R. P. Agarwal, M. A. El-Gebeily, and D. O’Regan, "Generalized contractions in partially ordered metric spaces," Applicable Analysis, vol. 87, no. 1, pp. 109-116, 2008.

[10] M. Turinici, "Ran-Reurings theorems in ordered metric spaces," The Journal of the Indian Mathematical Society. New Series, vol. 78, no. 1-4, pp. 207-214, 2011.

[11] V. Pragadeeswarar and M. Marudai, "Best proximity points: approximation and optimization in partially ordered metric spaces," Optimization Letters, 2013. 


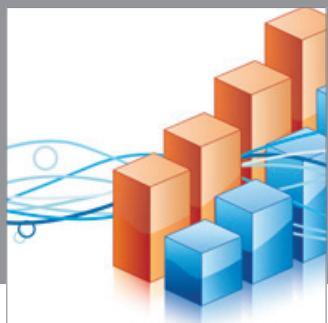

Advances in

Operations Research

mansans

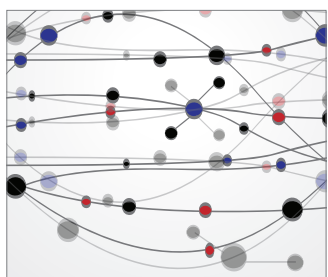

The Scientific World Journal
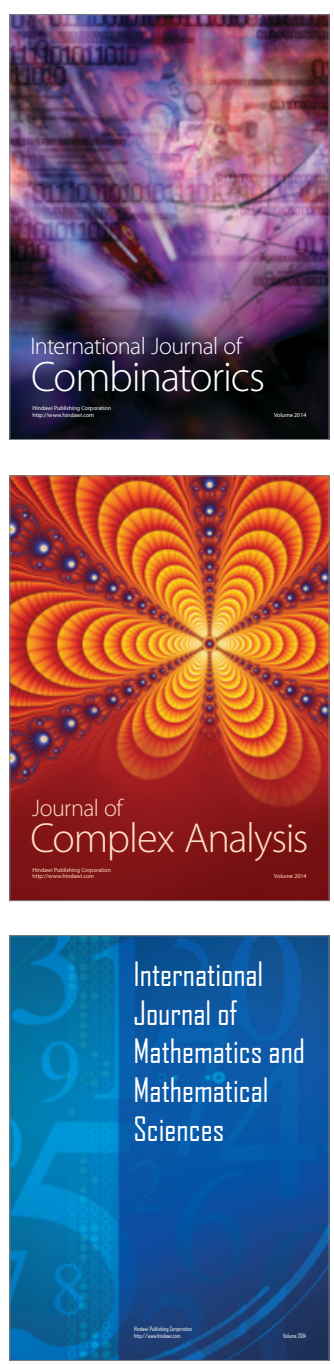
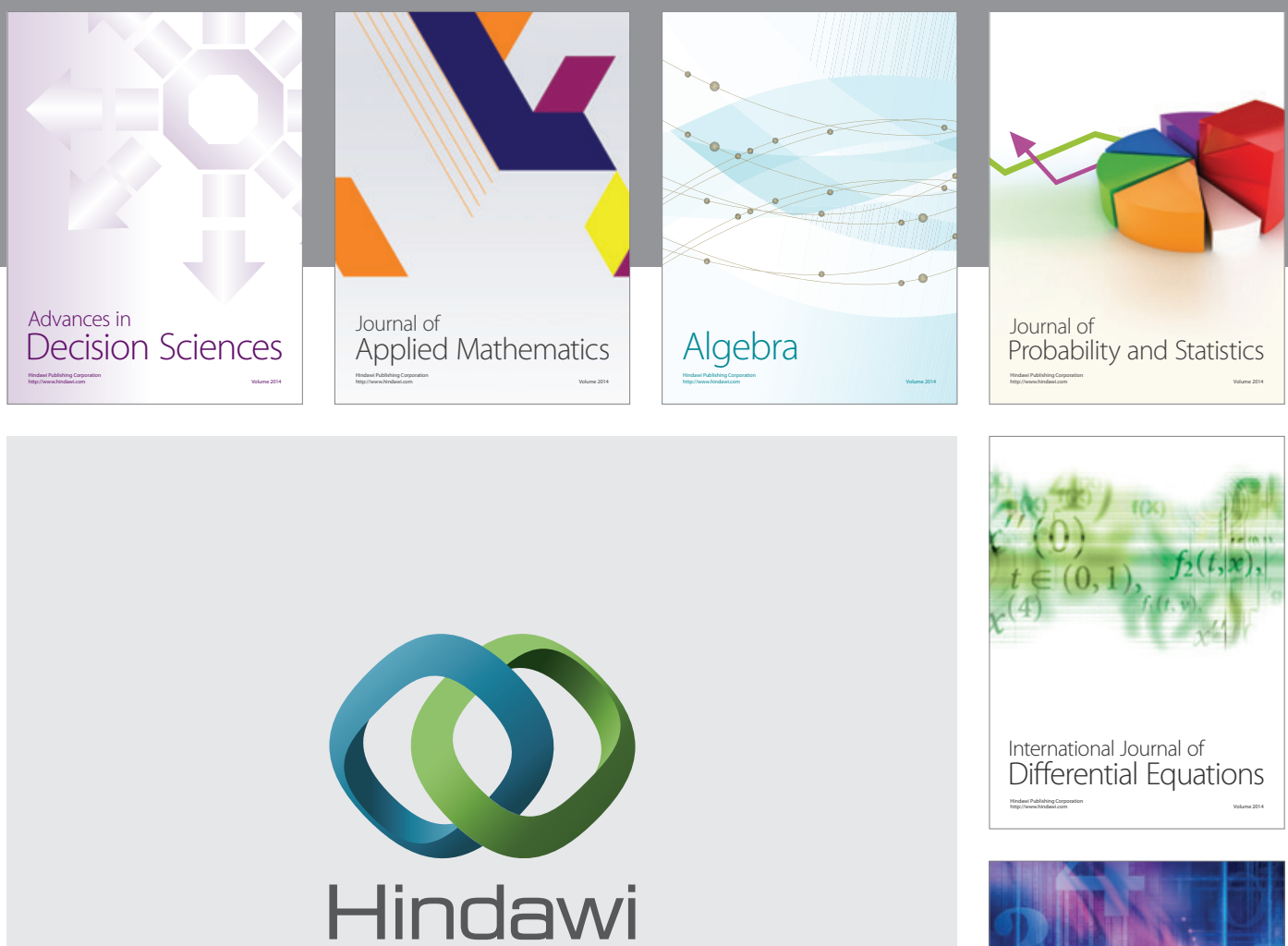

Submit your manuscripts at http://www.hindawi.com
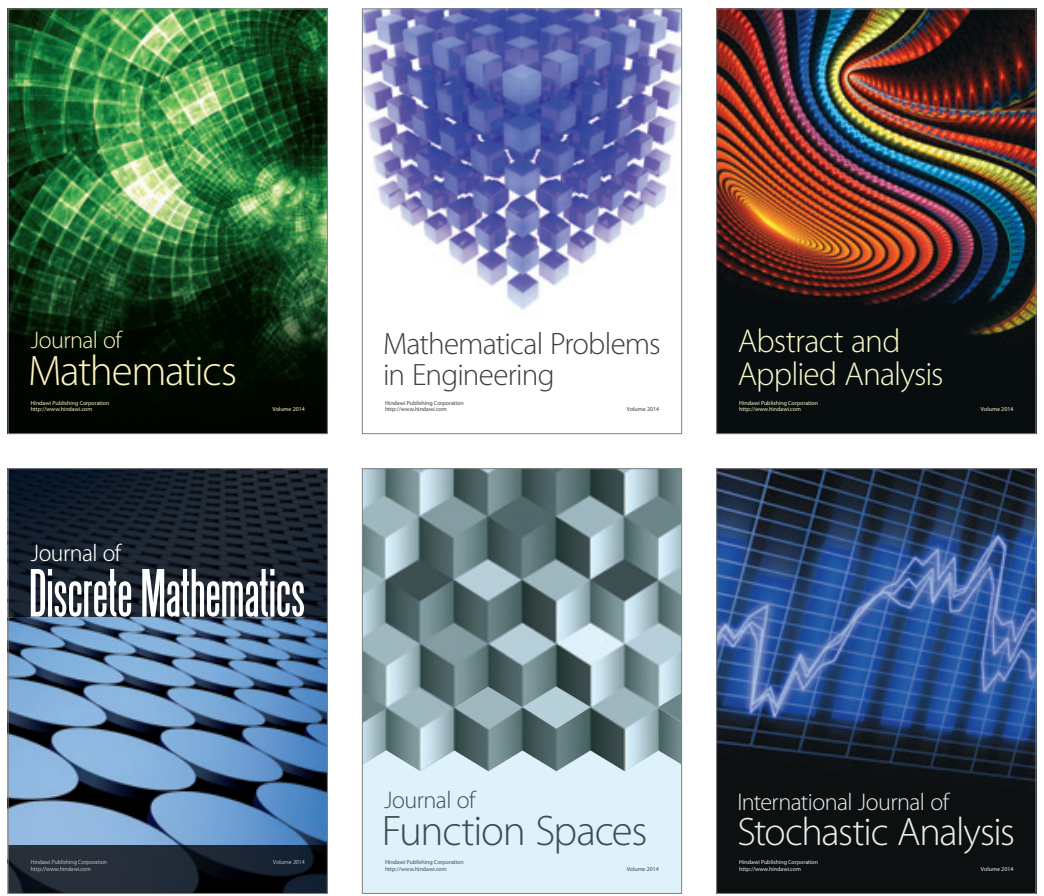

Journal of

Function Spaces

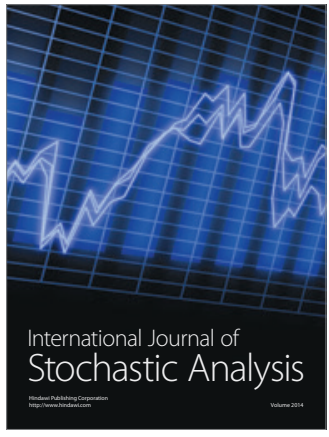

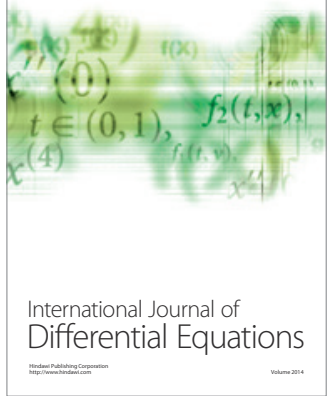
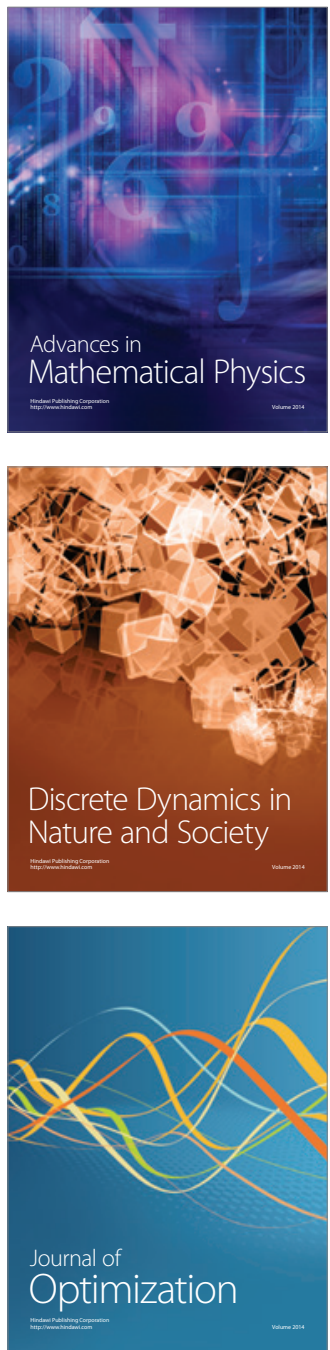